\title{
A semidefinite representation for some minimum cardinality problems
}

\author{
Alexandre d'Aspremont*
}

November 16, 2018

\begin{abstract}
Using techniques developed in Las02, we show that some minimum cardinality problems subject to linear inequalities can be represented as finite sequences of semidefinite programs. In particular, we provide a semidefinite representation of the minimum rank problem on positive semidefinite matrices. We also use this technique to cast the problem of finding convex lower bounds on the objective as a semidefinite program.

Keywords: semidefinite programming, sum of squares, rank minimization, $\mathbb{K}$ moment problem, Lagrangian relaxation.
\end{abstract}

\section{Notation}

We note $\mathbf{R}\left[x_{1}, \ldots, x_{n}\right]$ (or $\mathbf{R}[x]$ when there is no ambiguity) the ring of multivariate polynomials $p(x)=p\left(x_{1}, \ldots, x_{n}\right)$ on a variable $x \in \mathbf{R}^{n}$. We say that $p(x) \in \mathbf{R}[x]$ is $S O S$ when $p(x)$ is a sum of squares of polynomials in $\mathbf{R}[x]$. For $x \in \mathbf{R}^{n}, \operatorname{Card}(x)$ will be the cardinal of the set $\left\{i: x_{i} \neq 0\right\}$. We note $\mathbf{S}^{n}$ the set of $n \times n$ symmetric matrices. For multivariate polynomials, we adopt the multiindex notation $p(x)=\sum_{\alpha} p_{\alpha} x^{\alpha}$, where $x^{\alpha}:=x_{1}^{\alpha_{1}} x_{2}^{\alpha_{2}} \ldots x_{n}^{\alpha_{n}}$, and we note $d=\sum_{i=1}^{n} \alpha_{i}$ the degree of $p(x) . \mathbf{R}_{d}[x]$ is the set of polynomials of degree at most $d$. Finally, $C(p)$ will be the Newton polytope of the polynomial $p(x)$, with $C(p)=\operatorname{Co}\left(\left\{\alpha: p_{\alpha} \neq 0\right\}\right)$.

\section{Introduction}

Given a convex set $\mathcal{C} \subset \mathbf{R}^{n}$, we are interested in solving the following problem:

$$
\begin{array}{ll}
\operatorname{minimize} & \operatorname{Card}(x) \\
\text { subject to } & x \in \mathcal{C},
\end{array}
$$

in the particular case where $\mathcal{C}$ is described by a set of linear inequalities. Except in certain rare instances, this problem is very hard to solve (see [VB96]). Excellent heuristics exist however, a classical one (see [HHB99] for example) replacing the function $\mathbf{C a r d}(x)$ by $\|x\|_{1}$, its largest convex lower bound on the unit cube.

*Information Systems Laboratory \& Department of Management Science and Engineering, Terman bldg., Stanford University, Stanford, CA 94305-4023, USA. Internet: aspremon@stanford.edu 
A related problem is that of minimizing the rank of a p.s.d. matrix subject to LMI constraints:

$$
\begin{array}{ll}
\operatorname{minimize} & \operatorname{Rank}(X) \\
\text { subject to } & X \in \mathcal{C},
\end{array}
$$

where $\mathcal{C}$ is here an affine subset of the semidefinite cone (a LMI). In this case also, minimizing the nuclear norm $\|X\|_{*}$ of $X$ will produce excellent approximate solutions (see [FHB00]).

In this paper, using results by [Cas84], Sho87], Put93, CLR95], [Nes00, Las01], [PS01. and $\operatorname{Las02}$, we show that the $\operatorname{Min} \operatorname{Card}(x)$ and $\operatorname{Min} \operatorname{Rank}(X) \operatorname{problems}$ in (11) and (2) are equivalent to large semidefinite programs (see [NN94]). To be precise, based on a reformulation à la [Sho87] of problems (11) and (2), we use the technique in Las02] to produce a finite (possibly exponential) sequence of increasingly tighter semidefinite relaxations.

The rest of the paper is organized as follows. In section 2, we recall some key definitions and properties on semidefinite representability and the sum of squares representation of positive polynomials. We also summarize the application of these representations to semialgebraic problems. In section [3, we show that both the $\operatorname{Min} \operatorname{Card}(x)$ and the $\operatorname{Min} \operatorname{Rank}(X)$ problems are equivalent to large scale semidefinite programs. Based on the work by [Put93], [Nes00] and Las02] we explicitly construct in section 4 a sequence of semidefinite programs solving problems (11) and (2). We also show how the problem of finding optimal convex lower bounds on the objective function can be represented in a similar way. Finally, in section 5 , we discuss the complexity of these techniques.

\section{Sums of squares and semidefinite programming}

We quickly recall here some key definitions and properties linking semidefinite and semialgebraic problems.

Hilbert's $17^{\text {th }}$ problem (see [Rez96] for an overview), which asked if all positive polynomials could be written as sums of squares of other polynomials, has a positive answer in dimension one. Nes00] provides an efficient way of computing the $S O S$ representation of a given positive univariate polynomial in the following result from [Nes00].

Proposition 1 Let $p(x) \in \mathbf{R}[x]$ be a univariate polynomial of degree $d$. Then $p(x)$ for all $x \in \mathbf{R}$ iff there exists a matrix $X \in \mathbf{S}^{v}$ such that:

$$
p(x)=y_{x}^{T} X y_{x}, \text { with } X \succeq 0, \quad \text { for all } x \in \mathbf{R}
$$

with $v=\lceil d / 2\rceil$ and $y_{x}=\left(1, x, x^{2}, \ldots, x^{v}\right)$ is the list of univariate monomials up to degree $v$.

The coefficients of the polynomials in the representation are then computed as the eigenvectors of the matrix $X$.

In the general multivariate case, that representation property of positive polynomials is lost. It can be shown (see Ber80]) that the set of multivariate SOS polynomials is dense in the set of positive polynomials, but there are simple examples of positive polynomials that are not SOS. However, recent results in semialgebraic geometry (see Cas84, Sch91, Put93. or [PV99]) bridge the gap between positive and $S O S$ polynomials on compact semialgebraic sets. We cite here the result in [Put93]. Let $g_{k}(x) \in \mathbf{R}\left[x_{1}, \ldots, x_{n}\right]$ for $j=1, \ldots, r$, and we note 
$K$, the semialgebraic set defined by $K=\left\{x \in \mathbf{R}^{n}: g_{k}(x) \geq 0, k=1, \ldots, r\right\}$. We suppose that $K$ is compact and that there exists $u(x) \in \mathbf{R}\left[x_{1}, \ldots, x_{n}\right]$ such that $\{u(x) \geq 0\}$ is compact with

$$
u(x)=u_{0}(x)+\sum_{k=1}^{r} g_{k}(x) u_{k}(x), \quad \text { for all } x \in \mathbf{R}^{n}
$$

where the polynomials $u_{k}(x) \in \mathbf{R}\left[x_{1}, \ldots, x_{n}\right]$ are $S O S$ for $k=1, \ldots, r$. Under this assumption, we can represent all polynomials positive on $K$ using $S O S$ polynomials as in Put93 or PV99.

Proposition 2 Suppose (4) holds. A polynomial $p(x) \in \mathbf{R}\left[x_{1}, \ldots, x_{n}\right]$ is positive on $K$ iff:

$$
p(x)=q_{0}(x)+\sum_{k=1}^{r} g_{k}(x) q_{k}(x), \quad \text { for all } x \in \mathbf{R}^{n}
$$

where the polynomials $q_{k}(x) \in \mathbf{R}\left[x_{1}, \ldots, x_{n}\right]$ are $S O S$ for $k=0, \ldots, r$.

Now, as in Par00] or Las01, we can write the multivariate version of the relation (31) mapping $S O S$ polynomials to the semidefinite cone.

Proposition 3 Let $p(x) \in \mathbf{R}\left[x_{1}, \ldots, x_{n}\right]$ be a polynomial and $K$ a semialgebraic set defined by $K=\left\{x \in \mathbf{R}^{n}: g_{k}(x) \geq 0, k=1, \ldots, r\right\}$, satisfying assumption (4). Then $p(x) \geq 0$ on $K$ iff there is an integer $m \in \mathbf{Z}_{+}$and matrices $X_{k} \in \mathbf{S}^{N}$, with $X_{k} \succeq 0$ for $k=0, \ldots, r$ such that:

$$
p(x)=y_{x}^{T} X_{0} y_{x}+\sum_{k=1}^{r}\left(y_{x}^{T} X_{k} y_{x}\right) g_{k}(x), \quad \text { for all } x \in \mathbf{R}^{n},
$$

where $N=\left\lceil\left(\begin{array}{c}n+m-1 \\ m\end{array}\right) / 2\right\rceil$ and $y_{x}=\left(1, x_{1}, \ldots, x_{n}, x_{1}^{2}, x_{1} x_{2}, \ldots, x_{1} x_{n}, x_{2} x_{3}, \ldots, x_{n}^{2}, \ldots, x_{1}^{m}, \ldots, x_{n}^{m}\right)$ is the vector of all monomials in $\mathbf{R}\left[x_{1}, \ldots, x_{n}\right]$, up to degree $m$, listed in graded lexicographic order.

The result on polynomials above shows that testing the positivity of a multivariate polynomial on a semialgebraic set $K$ satisfying the assumption (41) can be cast as a semidefinite program. In general, the result in Las01] shows that all compact semialgebraic problems, i.e. problems seeking to minimize a polynomial over a compact semialgebraic set, are equivalent to large-scale semidefinite programs. This provides a positive answer in the compact multivariate case to all the open questions in $\$ 4.10 .2$ in BTN01. A converse result is also true (and much simpler). Because the positive semidefiniteness of a matrix is equivalent to that of all its principal minors, all semidefinite programs are semialgebraic programs, with additional convexity and invariance properties.

The central result of moment theory exploited in Las01 sets polynomial positivity problems and moment problems as duals (see e.g. [Ber80]). Let $s$ be a positive semidefinite sequence $s \in \mathbf{R}^{N}$, we have

$$
\begin{aligned}
& s \text { is p.s.d. } \\
& \left\langle s, p_{\alpha}\right\rangle \geq 0, \quad \text { for all } p(x) \in \mathbf{R}_{m}[x] \text { with } p(x) \text { SOS, }
\end{aligned}
$$


and

$$
\begin{gathered}
s \text { is a moment sequence } \\
\left\langle s, p_{\alpha}\right\rangle \geq 0, \quad \text { for all } p(x) \in \mathbf{R}_{m}[x] \text { with } p(x) \geq 0 \text { on } \mathbf{R}^{n},
\end{gathered}
$$

hence the cone of coefficients of $S O S$ polynomials and that of p.s.d. sequences are polar, and so are the cones of moment sequences and positive polynomials.

From [Put93] then, we know that the problem of testing if a sequence $y$ is the moment sequence of some measure $\mu$ with support in a compact semialgebraic set

$$
K=\left\{x \in \mathbf{R}^{n}: g_{k}(x) \geq 0, k=1, \ldots, r\right\}
$$

and the problem of representing positive polynomials on $K$ as $g_{k}(x)$ weighted sums of $S O S$ polynomials are dual of each other and both representable as linear matrix inequalities.

\section{Semidefinite representation of the $\operatorname{MinCard}(\mathrm{x})$ and MinRank(X) problems.}

As above $K$ is the semialgebraic set defined by $K=\left\{x \in \mathbf{R}^{n}: g_{k}(x) \geq 0, k=1, \ldots, r\right\}$ and we assume that (41) holds. Let $p(x) \in \mathbf{R}\left[x_{1}, \ldots, x_{n}\right]$ and as $K$ is compact, we also note $t^{*}=\min _{K} p(x)$. Of course, $p(x)-t^{*} \geq 0$ on $K$, hence there are $S O S$ polynomials $q_{k}(x) \in$ $\mathbf{R}\left[x_{1}, \ldots, x_{n}\right]$ for $k=1, \ldots, r$ such that (5) holds for $p(x)-t^{*}$ on $K$. We first show that the Min $\operatorname{Card}(x)$ problem can be cast as a semialgebraic program, hence a semidefinite program, using the results from section 2 .

Proposition 4 Let $A \in \mathbf{R}^{m \times n}$ and $b \in \mathbf{R}^{m}$. There are polynomials $g_{k}(x) \in \mathbf{R}\left[x_{1}, \ldots, x_{n}\right]$, for $k=0, \ldots, r$ such that the optimum values of:

$$
\begin{array}{ll}
\text { minimize } & \operatorname{Card}(x) \\
\text { subject to } & A x \geq b,
\end{array}
$$

and

$$
\begin{array}{ll}
\text { minimize } & g_{0}(x) \\
\text { subject to } & g_{k}(x) \succeq 0, \quad \text { for } i=1, \ldots, r,
\end{array}
$$

are equal.

Proof. First, as in Sho87] we notice that:

$$
\begin{aligned}
\operatorname{Card}(x)=\min & \sum_{i=1}^{n} v_{i} \\
\text { s.t. } & \left(v_{i}-1\right) x_{i}=0 \\
& v_{i} \geq 0, \quad \text { for } i=1, \ldots, n,
\end{aligned}
$$

hence the $\operatorname{Min} \operatorname{Card}(x)$ problem in (7) can be written:

$$
\begin{aligned}
\operatorname{Min} \operatorname{Card}(x) \equiv \min . & \sum_{i=1}^{n} v_{i} \\
\text { s.t. } & \left(v_{i}-1\right) x_{i}=0 \\
& v_{i} \geq 0, \quad \text { for } i=1, \ldots, n \\
& A x \geq b .
\end{aligned}
$$

which is a semialgebraic problem. 
We now show a similar result on the $\operatorname{Min} \operatorname{Rank}(X)$, a minimum cardinality problem on the eigenvalues of the matrix $X$.

Proposition 5 Let $A_{i} \in \mathbf{S}^{n}$, for $i=1, \ldots, p$ and $b \in \mathbf{R}^{p}$. There are polynomials $g_{k}(x) \in$ $\mathbf{R}\left[x_{1}, \ldots, x_{n}\right]$, for $k=0, \ldots, r$ such that the optimum values of:

$$
\begin{array}{ll}
\text { minimize } & \operatorname{Rank}(X) \\
\text { subject to } & \operatorname{Tr}\left(A_{i} X\right)=b_{i}, \quad \text { for } i=1, \ldots, p \\
& X \succeq 0
\end{array}
$$

and

$$
\begin{array}{ll}
\text { minimize } & g_{0}(x) \\
\text { subject to } & g_{k}(x) \succeq 0, \quad \text { for } i=1, \ldots, M
\end{array}
$$

are equal.

Proof. We note $\left(\lambda_{1}, \ldots, \lambda_{n}\right)$ the eigenvalues of the matrix $X \succeq 0$ and

$$
\sigma_{k}(X)=\sum_{\alpha \subset\{1, \ldots, n\},|\alpha|=k} \lambda_{\alpha}
$$

the symmetric functions. We note $\chi_{t}(X)$ the characteristic polynomial of the matrix $X$, with $\chi_{t}(X)=\sum_{i=1}^{n}(-1)^{i} \sigma_{i}(X) t^{i}$. Because the matrix $X$ is semidefinite positive, we have $\sigma_{k}(X)=0$ iff $\operatorname{Rank}(X)<k$, hence the $\operatorname{Min} \operatorname{Rank}(X)$ problem can be expressed as a minimum cardinality problem on the coefficients of the characteristic polynomial. With

$$
\begin{aligned}
\operatorname{Rank}(X)=\min & v_{i} \\
\text { s.t. } & \sigma_{i}(X)\left(v_{i}-1\right)=0 \\
& v_{i} \geq 0, \quad \text { for } i=1, \ldots, n,
\end{aligned}
$$

we enforce the remaining constraints to get the following representation of $\operatorname{Min} \operatorname{Rank}(X)$ :

$$
\begin{array}{ll}
\operatorname{minimize} & \sum_{i=1}^{n} v_{i} \\
\text { s.t. } & \left(v_{i}-1\right) \sigma_{i}(X)=0 \\
& v_{i} \geq 0, \quad \text { for } i=1, \ldots, n \\
& \operatorname{Tr}\left(A_{i} X\right)=b_{i}, \quad \text { for } i=1, \ldots, p \\
& d_{I}(X) \geq 0, \quad \text { for } I \subset\{1, \ldots, n\},
\end{array}
$$

where $d_{I}(X)$ is the principal minor with index set $I \subset\{1, \ldots, n\}$. This is a semialgebraic program in the coefficients of the matrix $X$.

These two results together with the results cited in section 2 show that the two problems considered are equivalent to very large scale semidefinite programs.

\section{Semidefinite relaxations}

In practice, the exact representations obtained in the last section can be exponentially large and in general, we cannot expect these problems to be tractable. Hence, the central contribution of these representations is not to reduce the complexity of these problems, but to 
provide a sequence of successively sharper relaxations covering the entire complexity spectrum, thus allowing the complexity/sharpness tradeoff to be tuned. This is what we intend to describe in this section.

We begin by recalling the construction of moment matrices as detailed in [CF00, Las01] and Las02]. Again, we let $y_{x}=\left(1, x_{1}, \ldots, x_{n}, x_{1}^{2}, x_{1} x_{2}, \ldots, x_{1} x_{n}, x_{2} x_{3}, \ldots, x_{n}^{2}, \ldots, x_{1}^{m}, \ldots, x_{n}^{m}\right)$ be the vector of all monomials in $\mathbf{R}\left[x_{1}, \ldots, x_{n}\right]$, up to degree $m$, listed in increasing graded lexicographic order. We note $s(m)$ the size of the vector $y_{x}$. Let $y \in \mathbf{R}^{s(2 m)}$ be the vector of moments (indexed according to $y_{x}$ ) of some probability measure $\mu$ with support $K=$ $\left\{x \in \mathbf{R}^{n}: g(x) \geq 0\right\}$, we note $M_{m}(y) \in \mathbf{S}^{s(m)}$, for the moment matrix defined by

$$
M_{m}(y)_{i, j}=\int_{K}\left(y_{x}\right)_{i}\left(y_{x}\right)_{j} \mu(d x), \quad \text { for } i, j=1, \ldots, s(m)
$$

i.e. the (symmetric) matrix of moments with rows and columns indexed as in $y_{x}$. We note $\beta(i)$ the exponent of the monomial $\left(y_{x}\right)_{i}$ and conversely, we note $i(\beta)$ the index of the monomial $x^{\beta}$ in $y_{x}$. For a given moment vector $y \in \mathbf{R}^{s(m)}$ ordered as in (41), the first row and columns of the matrix $M_{m}(y)$ are then equal to $y$. The rest of the matrix is then constructed following:

$$
M_{m}(y)_{i, j}=y_{\alpha+\beta} \text { if } M_{m}(y)_{1, i}=y_{\alpha} \text { and } M_{m}(y)_{j, 1}=y_{\beta} .
$$

Similarly, let $g(x) \in \mathbf{R}\left[x_{1}, \ldots, x_{n}\right]$, we derive the moment matrix for the measure $g(x) \mu(d x)$ on $K$ (called the localizing matrix), noted $M_{m}(g y) \in \mathbf{S}^{s(m)}$, from the matrix of moments $M_{m}(y)$ by:

$$
M_{m}(g y)_{i, j}=\int_{K}\left(y_{x}\right)_{i}\left(y_{x}\right)_{j} g(x) \mu(d x)
$$

for $i, j=1, \ldots, s(m)$. The coefficients of the matrix $M_{m}(g y)$ are then given by:

$$
M_{m}(g y)_{i, j}=\sum_{\alpha} g_{\alpha} M_{m}(y)_{i(\beta(i)+\beta(j)+\alpha)}
$$

We can remark as in Las01 that if the measure $\mu$ has its support included in $K=$ $\left\{x \in \mathbf{R}^{n}: g(x) \geq 0\right\}$, then for all coefficient vectors $v \in \mathbf{R}^{s(m)}$ :

$$
\left\langle v, M_{m}(g y) v\right\rangle=\int_{K} v(x)^{2} g(x) \mu(d x) \geq 0
$$

hence $M_{m}(g y) \succeq 0$.

In dimension one, for a given vector $y \in \mathbf{R}^{s(2 m)}, M_{m}(y) \succeq 0$ (which is a LMI) is also a sufficient condition in order for $y$ to the moment sequence of a probability measure. In $\mathbf{R}^{n}$, this equivalence does not hold in general. The compact semialgebraic case is called the K-moment problem and is dual to the compact $S O S$ problem in (66). Following [Las01], we now exploit this duality to compute a sequence of semidefinite relaxations for the Min Card $(x)$ and $\operatorname{Min} \operatorname{Rank}(X)$ problems. 


\subsection{The MinCard(x) problem}

In section 3, we saw that the optimum value of the Min $\operatorname{Card}(x)$ problem can be computed as the optimum value of the semialgebraic program:

$$
\begin{array}{ll}
\min . & \sum_{i=1}^{n} v_{i} \\
\text { s.t. } & \left(v_{i}-1\right) x_{i}=0 \\
& v_{i} \geq 0, \quad \text { for } i=1, \ldots, n \\
& a_{j}^{T} x \geq b_{j}, \quad \text { for } j=1, \ldots, m .
\end{array}
$$

As in Las01, to ensure compactness, we impose the additional constraint $x_{1}^{2}+\ldots+x_{n}^{2} \leq \alpha$ for some constant $\alpha>1$. It is easy to check that the program above, together with this additional bound on the feasible set, satisfies the constraints qualification assumption (41). For $N \geq 1$, a lower bound $l_{N}$ on the optimal value of the above problem is then computed as:

$$
\begin{aligned}
l_{N}:=\inf & \sum_{i=1}^{n} y_{i} \\
\text { s.t. } & M_{N}(y) \succeq 0 \\
& M_{N-1}\left(x_{i}\left(v_{i}-1\right) y\right)=0 \\
& M_{N-1}\left(\left(\alpha-x^{T} x-v^{T} v\right) y\right) \succeq 0 \\
& M_{N-1}\left(v_{i} y\right) \succeq 0, \quad \text { for } i=1, \ldots, n \\
& M_{N-1}\left(\left(a_{j}^{T} x-b_{j}\right) y\right) \succeq 0, \quad \text { for } j=1, \ldots, m,
\end{aligned}
$$

in the variable $y \in \mathbf{R}^{2 n}$. Theorem 3.2 in Las02 then states that there exists some $N^{*}$ such that

$$
l_{N}=\operatorname{Min} \operatorname{Card}(x), \quad \text { for all } N \geq N^{*},
$$

and the optimum is achieved whenever the rank of the matrices $M_{N}((\ldots) y)$ stabilizes.

\subsection{The MinRank(X) problem}

In section 3, for $X \in \mathbf{S}^{n}$, we saw that the optimum of the $\operatorname{Min} \operatorname{Rank}(X)$ problem can be computed as the optimum value of the semialgebraic program:

$$
\begin{array}{ll}
\min . & \sum_{i=1}^{n} v_{i} \\
\text { s.t. } & \left(v_{i}-1\right) \sigma_{i}(X)=0 \\
& v_{i} \geq 0, \quad \text { for } i=1, \ldots, n \\
& \operatorname{Tr}\left(A_{j} X\right)=b_{j}, \quad \text { for } j=1, \ldots, p \\
& d_{I}(X) \geq 0, \quad \text { for } I \subset[1, n],
\end{array}
$$

To further simplify this program, we can substitute to the $2^{n}$ constraints on the principal minors a more economical semialgebraic constraint. The modified program then reads:

$$
\begin{array}{ll}
\min . & \sum_{i=1}^{n} v_{i} \\
\text { s.t. } & \left(v_{i}-1\right) \sigma_{i}(X)=0 \\
& v_{i} \geq 0, \quad \text { for } i=1, \ldots, n \\
& \operatorname{Tr}\left(A_{i} X\right)=b_{i}, \quad \text { for } i=1, \ldots, p \\
& u^{T} X u \geq 0, \quad \text { for } u \in \mathbf{R}^{n}
\end{array}
$$


and again, to ensure compactness, we impose $X^{T} X+v^{T} v+u^{T} u \leq \alpha$ for some constant $\alpha>1$. If we set the variable $x=(u, X, v)$, for $N \geq\left\lceil\frac{n+1}{2}\right\rceil$, a lower bound $l_{N}$ on the optimal value of the above problem is computed as:

$$
\begin{aligned}
l_{N}:=\inf & \sum_{i=1}^{n} u_{i} \\
\text { s.t. } & M_{N}(y) \succeq 0 \\
& M_{N-\left\lceil\frac{i+1}{2}\right\rceil}\left(\left(v_{i}-1\right) \sigma_{i}(X) y\right)=0 \\
& M_{N-1}\left(v_{i} y\right) \succeq 0 \\
& M_{N-1}\left(\left(T r\left(A_{i} X\right)-b_{i}\right) y\right)=0 \\
& M_{N-1}\left(\left(\alpha-X^{T} X+v^{T} v+u^{T} u\right) y\right) \succeq 0 \\
& M_{N-2}\left(\left(u^{T} X u\right) y\right) \succeq 0
\end{aligned}
$$

for $i=1, \ldots, n$ and $j=1, \ldots, p$, in the variable $y \in \mathbf{R}^{2 n}$, where the matrices $M(q(x) y)$ are computed as in (11). Theorem 3.2 in Las02 then states that there exists some $N^{*}$ such that

$$
l_{N}=\operatorname{Min} \operatorname{Rank}(X), \quad \text { for all } N \geq N^{*},
$$

and the optimum is reached whenever the rank of the matrices $M_{N}((\ldots) y)$ stabilizes. Alternatively, one could use the fact that if we note $\chi_{t}(X)$ the characteristic polynomial of $X$, then $X \succeq 0$ is equivalent to $\chi_{-t^{2}}(X)$ being $S O S$ as a univariate polynomial in $t$.

\subsection{Convex envelope}

Suppose that instead of having only one $\operatorname{Min} \operatorname{Card}(x)$ or $\operatorname{Min} \operatorname{Rank}(X)$ problem to solve, we need to solve a (long) sequence of these problems with only some variation in the constraints. Here, instead of computing an exact relaxation for every instance of the problem, we are interested in finding an efficient heuristic method for approximating the solution to all the problems to be solved. The complexity of the first "bound design" program will be high, but that of the subsequent programs will then be much lower. The heuristics in FHB00 replaced the $\operatorname{Card}(x)$ (resp. $\operatorname{Rank}(X))$ functions by their convex envelope on the sets $0 \leq x \leq 1$ (resp. $0 \preceq X \preceq I$ ), i.e. the largest convex function $f(x)$ such that $f(x) \leq \operatorname{Card}(x)$ if $0 \leq x \leq 1$ (resp. $f(X) \leq \operatorname{Rank}(X)$ if $0 \preceq X \preceq I$ ). In this section, we extend these bounds to semialgebraic sets with more complex shapes.

Of course, a function and its convex envelope share the same global minimum, so solving for this optimal lower bound is at least as hard as finding the global minimum. Here however we look for a convex lower bound for the problem in (17) inside the set of polynomials of degree at most $d$. This becomes a semialgebraic program:

$$
\begin{array}{ll}
\text { maximize } & \int_{[0,1]^{n}} p(x) d x \\
\text { subject to } & t-p(x) \geq 0 \text { on } K \\
& \sum_{i=1}^{n} v_{i}-p(x) \geq 0 \text { on } K \\
& u^{T} \nabla^{2} p(x) u \geq 0 \text { on }\|u\|^{2}=1
\end{array}
$$

in the coefficients $p_{\alpha}$ of the polynomial $p(x) \in \mathbf{R}_{d}\left[x_{1}, \ldots, x_{n}\right]$, where $K$ is the compact semialgebraic set given by:

$$
\begin{aligned}
K=(v, x) \in \mathbf{R}^{2 n}: & \left(v_{i}-1\right) x_{i}=0 \\
& A x-b \geq 0 \\
& x, v \geq 0
\end{aligned}
$$


Again, this can be cast as a LMI using the technique in Las02]. We notice that the $l_{1}$ heuristic is a particular case when the constraint $A x-b \geq 0$ is dropped.

\section{Complexity}

Of course, the two semidefinite programs detailed in the last section are far from tractable if the dimension $n$ and the relaxation order $N$ grow beyond textbook example sizes. The Min $\operatorname{Card}(x)$ problem is equivalent to solving $2^{n}$ linear programs, so it is right to ask whether the programs above provide any benefit over, for example, branch-and-bound methods?

Even if these two methodologies have similar worst-case complexities, the semidefinite relaxations in (13) and (14) do sometimes produce the global optimum for low order $N$ (see Las02] ) and because the objective is integer valued here, they only need to be solved up to an absolute precision of $1 / 2$. We quickly detail below some other possible simplifications.

But the results above have to be considered first as representations, providing an insight of the relative complexity of minimum cardinality problems versus that of tractable convex optimization problems.

\subsection{Structure, sparsity and symmetry}

The first element that can be used to simplify the programs in (13) and (14) is structure. In Las02] for example, the constraints $x \in\{-1,1\}$, that translate into $x^{2}-x=0$ and $M_{m}\left(\left(x^{2}-x\right) y\right)=0$ also imply that the variables $y_{\alpha}$, for $\alpha \in \mathbf{Z}_{+}^{n}$, can be replaced by $y_{\mathbf{C a r d}(\alpha)}$ in the program. Secondly, if the constraints in (13) and (14) include some symmetry, we could use the results in Gat00, and GP02 to preprocess and simplify the original semialgebraic program. The simplest example of these symmetries is of course when the constraints are invariant with respect to a change of basis, in which case $\operatorname{Min} \operatorname{Rank}(X)$ reduces to a $\operatorname{Min} \operatorname{Card}(x)$ problem and as in GP02] p. 31, the asymptotic complexity goes from being exponential in $n$ to being exponential in $\sqrt{n}$.

In general, the complexity of algorithms in semialgebraic geometry grows at least exponentially with the dimension. Efficiency is then very often measured by the ability of a method to maintain sparsity. Some results on Newton polytopes and SOS polynomials can then be used to efficiently handle sparsity in the SOS representations. In particular, a result of [Rez78] shows that if $p(x), h_{i}(x) \in \mathbf{R}[x]$, for $i=1, \ldots, r$, with $p(x)=\sum_{i=1}^{r} h_{i}^{2}(x)$, then $C\left(h_{i}\right) \subseteq \frac{1}{2} C(p)$. That result however does not hold as is for the representation in (15).

Finally, a lower bound on the optimal value can be obtained by simply dropping some of the constraints in (13) and (14).

\section{Conclusion}

One of the central contributions of semidefinite programs to the optimization toolbox is their ability to efficiently solve a wide class of convex eigenvalue problems. In this work, we have illustrated how the method described in [Las01] for solving semialgebraic programs, by lifting them to semidefinite programs, can also be used to represent some semialgebraic eigenvalue problems and convex envelope relaxations. This contribution is centered around 
semidefinite representations and the insight they can provide on the theoretical complexity

of these problems. Wether or not they also improve the practical complexity of computing relaxations to these problems remains to be explored.

\section{References}

[Ber80] C. Berg, The multidimensional moment problem and semi-groups, Moments in Mathematics (H.J. Landau, ed.), AMS, Providence, RI, 1980, pp. 110-124.

[BTN01] A. Ben-Tal and A. Nemirovski, Lectures on modern convex optimization : analysis, algorithms, and engineering applications, MPS-SIAM series on optimization, Society for Industrial and Applied Mathematics : Mathematical Programming Society, Philadelphia, PA, 2001.

[Cas84] Gilles Cassier, Problème de moments sur un compact de $R^{n}$ et décomposition de polynômes à plusieurs variables, Journal of Functional analysis 58 (1984), 254-266.

[CF00] R. Curto and A. Fialkow, The truncated complex K-moment problem, Transactions of the American mathematical society 352 (2000), no. 6, 2825-2855.

[CLR95] M. D. Choi, T. Y. Lam, and B. Reznick, Sums of squares of real polynomials, Proceedings of Symposia in Pure Mathematics 58 (1995), no. 2, 103-126.

[FHB00] M. Fazel, H. Hindi, and S. Boyd, A rank minimization heuristic with application to minimum order system approximation., Working paper. American Control Conference, September 2000 (2000).

[Gat00] Karin Gatermann, Computer algebra methods for equivariant dynamical systems, Lecture notes in mathematics, vol. 1728, Springer-Verlag, 2000.

[GP02] Karin Gatermann and P. Parillo, Symmetry groups, semidefinite programs, and sums of squares, Tech. Report arXiv: math.AC/0211450, ETH Zurich, 2002.

[HHB99] A. Hassibi, J. How, and S. Boyd, Low-authority controller design via convex optimization, AIAA Journal of Guidance, Control and Dynamics 22 (1999), no. 6, 862-872.

[Las01] J. B. Lasserre, Global optimization with polynomials and the problem of moments, SIAM Journal on Optimization 11 (2001), no. 3, 796-817.

[Las02] _ An explicit equivalent positive semidefinite program for nonlinear 0-1 programs, SIAM Journal on Optimization 12 (2002), no. 3, 756-769.

[Mot65] T.S. Motzkin, The arithmetic-geometric inequality, Inequalities Symposium (Wright-Patterson AFB) (O. Shisha, ed.), Academic Press, 1965.

[Nes00] I. Nesterov, Squared functional systems and optimization problems, Tech. Report 1472, CORE reprints, 2000. 
[NN94] I. Nesterov and A. Nemirovskii, Interior-point polynomial algorithms in convex programming, Society for Industrial and Applied Mathematics, Philadelphia, 1994.

[Par00] P. Parillo, Structured semidefinite programs and semialgebraic geometry methods in robustness and optimization, Ph.D. thesis, California Institute of Technology, 2000 .

[PS01] P. Parillo and Bernd Sturmfels, Minimizing polynomial functions, Tech. Report arXiv: math.OC/0103170, DIMACS, Rutgers University, 2001.

[Put93] M. Putinar, Positive polynomials on compact semi-algebraic sets, Indiana University Mathematics Journal 42 (1993), no. 3, 969-984.

[PV99] M. Putinar and F.-H. Vasilescu, Solving moment problems by dimensional extension, Annals of Mathematics 149 (1999), 1087-1107.

[Rez78] B. Reznick, Extremal psd forms with few terms, Duke mathematical journal 45 (1978), 363-374.

[Rez96] - Some concrete aspects of hilbert's 17th problem, Tech. Report 56, Séminaire de structures algébriques ordonnées: Equipe de logique mathématique Prépublications (F. Delon, M. Dickmann, D. Gondard, eds.), Publ. Math. Univ. Paris VII., 1996.

[Sch91] K. Schmüdgen, The K-moment problem for semi-algebraic sets, Mathematische Annalen 289 (1991), 203-206.

[Sho87] N.Z. Shor, Quadratic optimization problems, Soviet Journal of Computer and Systems Sciences 25 (1987), 1-11.

[VB96] L. Vandenberghe and S. Boyd, Semidefinite programming, SIAM Review 38 (1996), 49-95. 